\begin{tabular}{ccc} 
TẠP CHÍ KHOA HỌC ĐẠI HỌC TÂN TRÀO \\
ISSN: 2354 - 1431 \\
\hline
\end{tabular}

\title{
Mô đun xoắn trong mô hình q-state clock
}

\author{
Lương Minh Tuấn ${ }^{a b^{*}}$, Tạ Thành Long ${ }^{a}$, Dương Xuân Núi ${ }^{a c}$, \\ Đào Xuân Việt ${ }^{a}$, Nguyễ் Bá Đức ${ }^{d}$, Nguyễn Đức Trung Kiên ${ }^{a}$ \\ ${ }^{a}$ Truoòng Đại học Bách Khoa Hà Nội \\ ${ }^{b}$ Truoòng Đại họ Xây dựng, Hà Nội \\ ${ }^{c}$ Truờng Đại học Lâm nghiệp Hà Nội \\ ${ }^{d}$ Truờng Đại học Tân trào \\ *Email: tuanlm@nuce.edu.vn
}

\section{Thông tin bài viết}

Ngày nhận bài:

18/02/2020

Ngày duyệt đăng:

$10 / 3 / 2020$

Tù khóa:

Khoa hoc vật liệ; mô phỏng Monte Carlo; chuyển pha; vật liệu tì;; mô hinh q-state clock.

\section{Tóm tắt}

Chúng tôi nghiên cứu hiện tượng chuyển pha của mô hình $q$-state clock hai chiều bằng phương pháp mô phỏng Monte Carlo. Chúng tôi tính toán các đại lượng vật lý như là: nhiệt dung riêng, độ từ hoá và mô đun xoắn cho $q>5$ (cụ thể $q=6$ và 8 ). Nhiệt dung riêng và độ từ hóa chỉ ra mô hình có hai chuyển pha, bao gồm chuyển pha trên, $T_{2}$, giữa pha mất trật tự và pha giả trật tự và chuyển pha dưới, $T_{1}$, giữa pha giả trật tự và pha trật tự. Mô đun xoắn không những chỉ ra $T_{2}$ là chuyển pha Kosterlitz-Thouless mà còn có biểu hiện của chuyển pha $T_{1}$.

\section{Giới thiệu}

Mô hình 2D $q$-state clock được quan tâm nghiên cứu hiện tượng chuyển pha trong nhiều năm $[1,2,3,4$, 5]. Mô hình này là tổng quát hóa của mô hình $2 \mathrm{D}$ Ising, nhưng mô hình này trở thành mô hình $2 \mathrm{D} X Y$ khi số trạng thái $q$ tiến tới vô cùng. Mô hình $2 \mathrm{D}$ Ising có chuyển pha bậc hai giữa pha mất trật tự và pha trật tự. Mô hình 2D XY có chuyển pha Kosterlitz-Thouless (KT) giữa pha mất trật tự và pha giả trật tự [6]. Chuyển pha KT là một trong những khái niệm quan trọng trong vật lí thống kê vì nó có thể giải thích được các pha và chuyển pha trong lớp chuyển tiếp Josephson [7], màng tinh thể lỏng [8]. Thú vị ở chỗ, chuyển pha KT xuất hiện trong mô hình $q$-state clock tùy thuộc vào số trạng thái $q$ của véctơ spin.

Chuyển pha của mô hình $q$-state clock được nghiên cứu bằng lý thuyết [1] và khẳng định lai bằng mô phỏng số $[2,3,4]$. Các tác giả cùng đồng thuận cho rằng mô hình $q$-state clock chỉ có một chuyển pha bậc hai cho $q$ $<5$ và hai chuyển pha $\mathrm{KT}$ riêng biệt ở nhiệt độ xác định $T_{1}$ và $T_{2}\left(T_{2}>T_{1}\right)$ cho $q \geq 5$. Pha ở giữa các nhiệt độ này là pha giả trật tự giống như mô hình $\mathrm{XY}$ dưới nhiệt độ chuyển pha $\mathrm{KT}$, pha trên $T_{2}$ là pha mất trật tự, và pha dưới $T_{1}$ là pha trật tự [2].

Các nghiên cứu mô phỏng cho mô hình $q$-state clock sau này tập trung vào các vấn đề: i) tranh luận chuyển pha là $\mathrm{KT}$ hay không phải là $\mathrm{KT}$; ii) đại lượng vật lý nào mô tả được chuyển pha của mô hình này. Năm 1982, dựa trên biểu hiện của nhiệt dung riêng, độ từ hoá và độ từ thẩm Tobochnik và cộng sự [3] đã chứng minh mô hình $q$-state clock có hai chuyển pha KT riêng biệt với $q \geq 5$. Năm 2010, Baek và cộng sự [4] cho rằng mô đun xoắn của $q=5$ không tiến về 0 trong pha nhiệt độ cao, nên chuyển pha tại $T_{2}$ không phải là chuyển pha loại KT. Tác giả đã so sánh biểu hiện của mô đun xoắn giữa các trường hợp $q=3,4,5,6$ và khẳng định mô hình $q$-state clock chỉ có chuyển pha $\mathrm{KT}$ với $q \geq 6$. Ở đây, mô đun xoắn chỉ tính được nhiệt độ chuyển pha trên $T_{2}$ và không có biểu hiện của chuyển pha dưới $T_{1}$. 
Tuy nhiên, một số nghiên cứu năm 2013 [5] và 2014 [9], ủng hộ các kết luận trước đó là hai chuyển pha của mô hình $q$-state clock thuộc loại chuyển pha KT cho $q \geq 5$. Kumano và cộng sự cho rằng định nghĩa mô đun xoắn liên tục tính qua góc xoắn liên tục không phù hợp với hệ có spin gián đoạn, vì thế nhóm đưa ra định nghĩa mô đun xoắn mới (gọi là mô đun xoắn gián đoạn) được tính thông qua một góc xoắn rời rạc cho hệ spin gián đoạn. Các tác giả khẳng định mô đun xoắn rời rạc có biểu hiện đồng thời 2 chuyển pha $T_{1}$ và $T_{2}$ trong mô hình $q$-state clock. Sau đó, Baek và cộng sự đã công bố một nghiên cứu khác [10] trong đó tác giả tính toán mô đun xoắn liên tục cho mô hình $q$-state clock với điều kiện biên khác. Một lần nữa tác giả nhận định mô đun xoắn liên tục có thể mô tả chuyển pha trong $q$-state clock với $q>5$, nhưng vẫn chỉ biểu hiện cho chuyển pha $T_{2}$.

Từ đây đặt ra câu hỏi rằng: mô đun xoắn gián đoạn xác định được đồng thời nhiệt độ chuyển pha $T_{1}$ và $T_{2}$, vậy mô đun xoắn liên tục có xác định được đồng thời nhiệt độ chuyển pha $T_{1}$ và $T_{2}$ với $q>5$ hay không? Do đó, trong bài báo này chúng tôi nghiên cứu mô phỏng mô hình $q$-state clock tập trung tính toán các đại lượng là nhiệt dung riêng, độ từ hoá và mô đun xoắn liên tục cho $q>5$ (cụ thể $q=6$ và 8 ) bằng phương pháp mô phỏng Monte Carlo.

\section{Mô hình và phương pháp}

Mô hình 2D $q$-state clock (còn gọi là mô hình đồng hồ $q$ trạng thái), ở đây, vector spin có $q$ hướng ( $q=2,3$, $4, \ldots)$ các hướng này cách đều nhau một góc. Mô hình 2D $q$-state clock như một mô hình trung gian giữa hai mô hình $2 \mathrm{D}$ Ising $(q=2)$ và mô hình $2 \mathrm{D} \mathrm{XY}(q \rightarrow \infty)$. Mô hình 2D $q$-state clock được mô tả bởi hàm Hamilton sau [1, 2]:

$$
H=-J \sum_{\langle\mathrm{ij}\rangle} \cos \left(\theta_{i}-\theta_{j}\right)
$$

trong đó $\theta_{i}=\frac{2 \pi}{q} n$, với $n=0,1,2 \ldots$, là góc của spin thứ $i$ với trục $x, J=1$ là hằng số tương tác trao đổi giữa các spin.

Chúng tôi tiến hành mô phỏng $\mathrm{MC}$ cho mạng hai chiều hình vuông có kích thước $N=L \times L$, với $L=16$, $32,64,128$, và áp dụng điều kiện biên tuần hoàn. Để đưa hệ về trạng thái cân bằng chúng tôi sử dụng kết hợp giữa thuật toán Metropolis và thuật toán Wolff. Trong đó, một bước $\mathrm{MC}(\mathrm{MCs})$ được định nghĩa bằng 1 bước Wolff và 1 bước Metropolis. Điều kiện cân bằng đã được kiểm tra thông qua sự bằng nhau của nhiệt dung riêng tính theo công thức thống kê và theo biến thiên nhiệt độ. Chúng tôi chạy 5 mẫu để tính trung bình và sai số của các đại lượng vật lí bằng phương pháp Jackknife.

Các đại lượng vật lý thống kê được chúng tôi trình bày trong nghiên cứu này gồm:

Nhiệt dung riêng được định nghĩa theo biểu thức [3],

$$
C=\frac{1}{N^{2} k_{B} T^{2}}\left(\left\langle E^{2}\right\rangle-\langle E\rangle^{2}\right)=\frac{1}{k_{B} T^{2}}\left(\left\langle e^{2}\right\rangle-\langle e\rangle^{2}\right)
$$

trong đó $E=\langle H\rangle$ và $e=E / N$.

Độ từ hoá là một tham số biểu thị độ trật tự trong các mô hình từ, hay nói cách khác độ từ hóa của các mô hình từ chính là tham số trật tự, và được tính theo công thức như sau [9]:

$$
m=\frac{1}{N}\left[\left(\sum_{i} \cos \theta_{i}\right)^{2}+\left(\sum_{i} \sin \theta_{i}\right)^{2}\right]^{1 / 2}
$$

Mô đun xoắn liên tục được định nghĩa như sau [4]:

$$
\Upsilon=\langle e\rangle-\frac{1}{L^{2} T}\left\langle s^{2}\right\rangle
$$

Trong đó, $e=1 / L^{2} \sum_{\langle i j\rangle_{x}} \cos \left(\theta_{i}-\theta_{j}\right)$ là năng lượng liên kết giữa các spin lân cận theo trục $x$ tính cho một spin, $s=1 / L^{2} \sum_{\langle i j\rangle_{x}} \sin \left(\theta_{i}-\theta_{j}\right)$ đo sự thăng giáng (sự quay) của các spin theo trục $x$ với tổng lấy trên tất cả các liên kết theo một hướng và $\langle\cdots\rangle$ là trung bình nhiệt động lực học.

\section{Kết quả và thảo luận}

Trong mục này chúng tôi trình bày kết quả mô phỏng Monte Carlo và thảo luận đối với mô hình $q$ state clock cho mạng hai chiều hình vuông với $q>5$ (cụ thể $q=6$ và 8 ) cho các đại lượng vật lý nhiệt dung riêng $(C)$, độ từ hóa $(m)$ và mô đun xoắn $(Y)$.

\subsection{Nhiệt dung riêng}

Kết quả mô phỏng nhiệt dung riêng phụ thuộc nhiệt độ với các kích thước khác nhau $L=16,32,64$ và 128 cho hai trường hợp điển hình $q=6$ (hình $1 \mathrm{a}$ ) và $q=8$ (hình $1 \mathrm{~b}$ ) có hai đỉnh tương ứng với dấu hiệu của hai chuyển pha. Khi nhiệt độ giảm, kết quả mô phỏng nhiệt dung riêng cho cả hai trường hợp $(q=6$ và $q=8)$ của đỉnh thứ hai có hình dạng tù, phụ thuộc vào kích thước 
(khi kích thước tăng nhiệt dung riêng dịch về phía nhiệt độ thấp), đây là biểu hiện của chuyển pha KT tại nhiệt độ $T_{2}$ từ pha mất trật tự sang pha giả trật tự [11]. Tuy nhiên, đỉnh thứ nhất có biểu hiện dạng tù tại nhiệt độ $T_{1}$ nhưng không phụ thuộc kích thước (kích thước tăng đỉnh cố định mà không dịch về phía nhiệt độ thấp), điều này cho thấy khó phân loại được chuyển pha dựa vào biểu hiện của đỉnh này. Do đó, chúng tôi đi xem xét đại lượng độ từ hóa và mô đun xoắn để xác định loại chuyển pha cho hai trường hợp này, đặc biệt vùng nhiệt độ thấp.
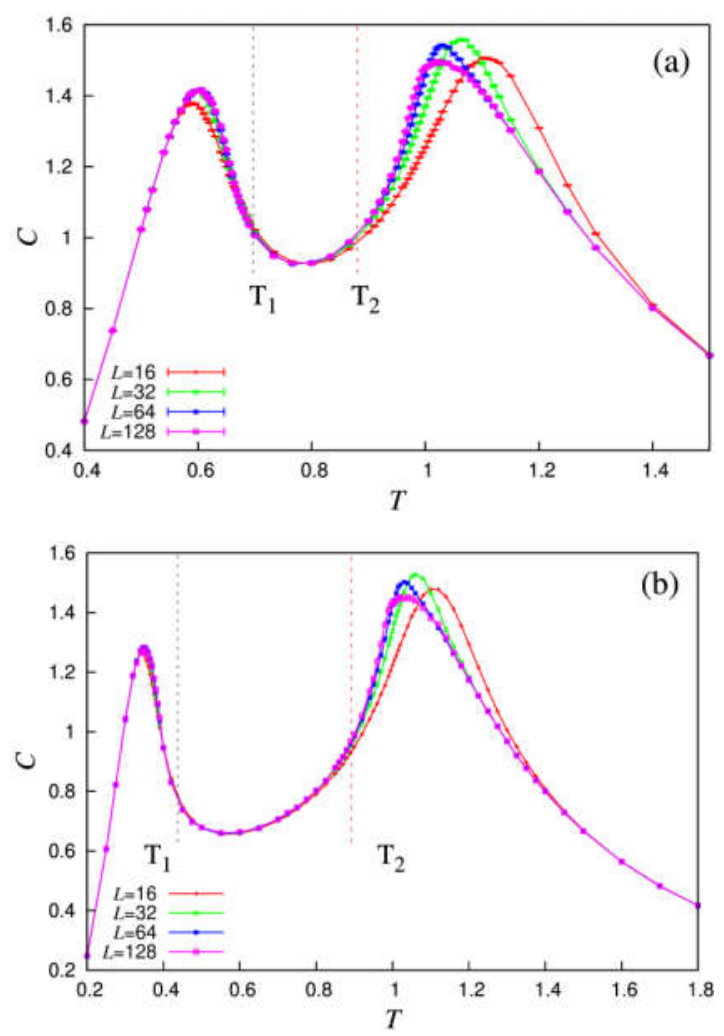

Hình 1: Sụ phu thuộc nhiệt độ của nhiệt dung riêng:

$$
\text { a) } q=6 \text { và b) } q=8 \text {. }
$$

\section{2. Độ tù̀ hoá}

Hình 2 biểu diễn độ từ hoá phụ thuộc vào nhiệt độ với các kích thước $L=16,32,64,128$. Trong cả hai trường hợp $q=6$ (hình $2 \mathrm{a}$ ) và $q=8$ (hình $2 \mathrm{~b}$ ), khi nhiệt độ giảm dần độ từ hóa có biểu hiện rõ ràng hai bước nhảy (đặc biệt với các kích thước mô phỏng lớn) tương ứng với hai chuyển pha. Xem xét kĩ hơn cho hai trường hợp này, chúng tôi thấy rằng bước nhảy ở gần nhiệt độ $T_{2}$ thể hiện chuyển pha từ pha mất trật tự nhiệt độ cao sang pha giả trật tự. Thật vậy ở vùng $T>T_{2}$ độ từ hoá bằng không thể hiện pha mất trật tự, còn trong vùng $T_{1}$ $<T<T_{2}$ độ từ hoá cũng có xu hướng tiến chậm về không khi kích thước $L \rightarrow \infty$ đây là dấu hiệu chỉ ra pha giả trật tự ở vùng nhiệt độ này. Còn đối với vùng nhiệt độ $T<T_{1}$ thì độ từ hoá có xu hướng tiến về giá trị hữu hạn với mọi kích thước, đây là biểu hiện của pha trật tự.
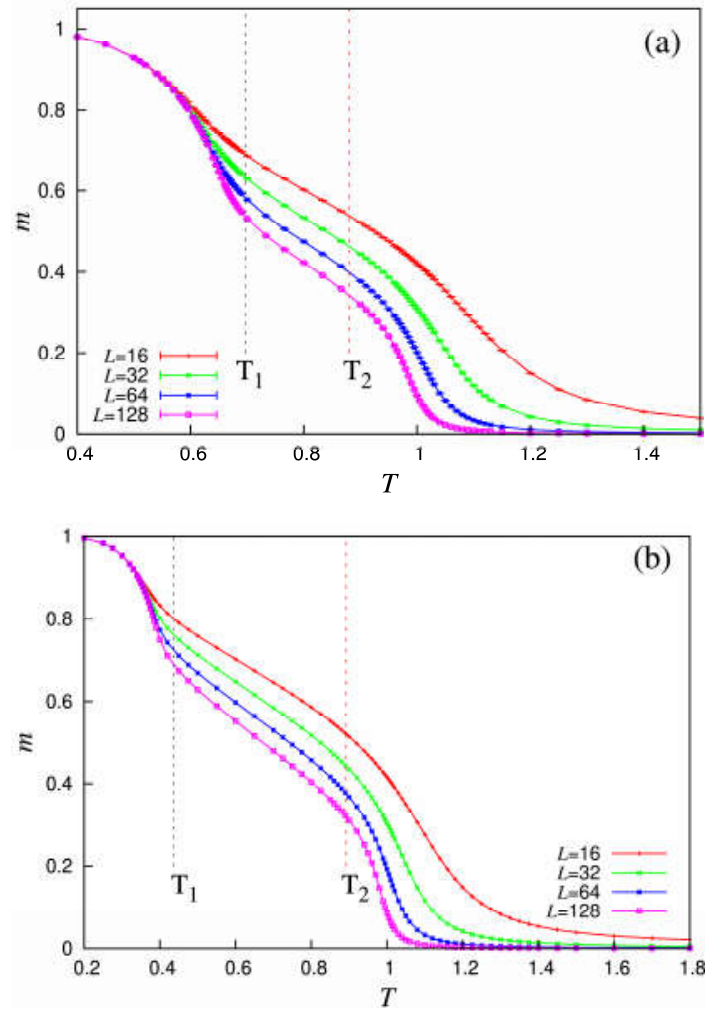

Hình 2: Sụ phụ thuộc nhiệt độ của độ tì hoá:

$$
\text { a) } q=6 \text { và b) } q=8 \text {. }
$$

\subsection{Mô đun xoắn}

Để làm sáng tỏ hơn cho nhận xét kể trên đối với độ từ hoá, cũng như phản biện lại những quan sát trước đây cho rằng ra mô đun xoắn liên tục chỉ biểu hiện chuyển pha $T_{2}$ trong nghiên cứu [4] và không sử dụng được đại lượng này trong nghiên cứu chuyển pha $[5,9]$ cho $q>5$. Chúng tôi đi khảo sát sự phụ thuộc vào nhiệt độ của mô đun xoắn (hình 3 ) cho các giá trị $q=6$ (hình $3 \mathrm{a})$ và 8 (hình $3 \mathrm{~b}$ ) với các kích thước $L=16,32,64$ và 128. Khi kích thước $L$ tăng, mô đun xoắn có biểu hiện bước nhảy tại $T_{2}$ và tiến về 0 ở vùng nhiệt độ $T>T_{2}$ [4], đây là biểu hiện của chuyển pha $\mathrm{KT}$ từ pha mất trật tự sang pha giả trật tự tại nhiệt độ $T_{2}$. Kết quả này phù hợp với các nghiên cứu trước $[4,5,9]$. Ngoài ra, khi quan sát $q=6,8$ ở vùng nhiệt độ thấp, chúng tôi, lần đầu tiên chỉ ra rằng $Y$ có biểu hiện điểm gẫy (đặc biệt biểu hiện này rõ ràng hơn khi $q$ lớn) gần $T_{1}$. Đây chính là dấu hiệu của chuyển pha $T_{1}$ và phù hợp với biểu hiện chuyển pha $T_{1}$ thông qua độ từ hóa. Vậy, mô đun xoắn liên tục, bản thân nó, có thể biểu hiện đồng thời hai chuyển pha trong mô hình $q$-state clock với $q>5$. 

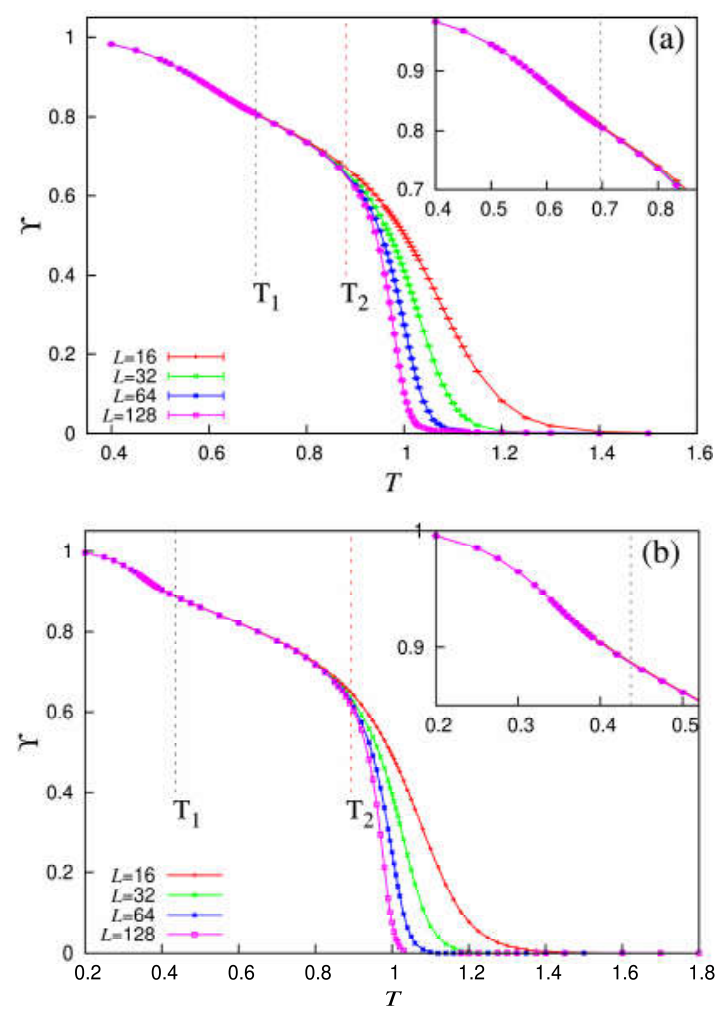

Hình 3: Sụ phu thuộc vào nhiệt độ của mô đun xoắn: a) $q=6$ và b) $q=8$.

\section{Kết luận}

Chúng tôi đã tiến hành nghiên cứu hiện tượng chuyển pha của mô hình $q$-state clock trong mạng hai chiều hình vuông cho $q>5$ bằng phương pháp mô phỏng Monte Carlo. Chúng tôi đã tính toán các đại lượng vật lý như là nhiệt dung riêng, độ từ hoá và mô đun xoắn. Nhiệt dung riêng có biểu hiện của hai chuyển pha nhưng không chỉ rõ bản chất chuyển pha vùng nhiệt độ thấp, độ từ hóa biểu hiện rõ ràng chuyển pha nhiệt độ thấp giữa pha giả trật tự và pha trật tự. Đặc biệt, lần đầu tiên chỉ ra mô đun xoắn có dấu hiệu chuyển pha giữa pha giả trật tự và pha trật tự.

\section{Lời cảm ơn}

Nghiên cứu này được tài trợ bởi Quỹ Phát triển khoa học và công nghệ Quốc gia (NAFOSTED) trong đề tài mã số 103.05-2019.44. Chuoong trình mô phỏng được thực hiện trên hệ máy tính của Viện Tiên tiến Khoa học và Công nghê,, Truờng Đại hoc Bách khoa Hà Nội.

\section{TÀI LIỆU THAM KHẢO}

1. J. V. Jose, L. P. Kadanoff, S. Kirkpatrick, and D. R.Nelson, "Renormalization, vortices, and symmetrybreaking perturbations in the two-dimensional planar model," Phys. Rev. B, vol. 16, p. 1217, 1977.

2. S. Elitzur, R. B. Pearson, and J. Shigemitsu, "Phase structure of discrete abelian spin and gauge systems," Phys. Rev. D, vol. 19, p. 3698, 1979.

3. J. Tobochnik, "Properties of the q-state clock model for q=4, 5, and 6," Phys. Rev. B, vol. 26, p. 6201, 1982.

4. S. K. Baek and P. Minnhagen, "Non-kosterlitzthouless transitions for the q-state clock models," Phys. Rev. E, vol. 82, p. 031102, 2010.

5. Y. Kumano, K. Hukushima, Y. Tomita, and M. Oshikawa, "Response to a twist in systems with zp symmetry: The two-dimensional p-state clock model," Phys. Rev. B, vol. 88, p. 104427, 2013.

6. J. M. Kosterlitz and D. J. Thouless, "Ordering, metastability and phase transitions in two-dimensional systems," Journal of Physics C: Solid State Physics, vol. 6 , p. 1181, 1973.

7. M. R. Beasley, J. E. Mooij, and T. P. Orlando, "Possibility of Vortex-Antivortex Pair Dissociation in Two-Dimensional Superconductors," Phys. Rev. Lett., vol. 42, p. 1175, 1979.

8. D. J. Bishop and J. D. Reppy, "Study of the superfluid transition in two-dimensional 4He films," Phys. Rev. B, vol. 22, p. 5171, 1980.

9. C. Chatelain, "Dmrg study of the berezinskiikosterlitz-thouless transitions of the $2 \mathrm{~d}$ five-state clock model," Journal of Statistical Mechanics: Theory and Experiment, vol. 11, p. 11022, 2014.

10. S. K. Baek, H. Mäkelä, P. Minnhagen, and B. J. Kim, "Residual discrete symmetry of the five-state clock model," Phys. Rev. E, vol. 88, p. 012125, 2013.

11. D B Carpenter and J T Chalker, "The phase diagram of a generalised XY model," J. Phys.: Condens. Matter, vol. 1, p. 4907, 1989. 


\section{Helicity modulus in a q-state clock model}

Luong Minh Tuan, Ta Thanh Long, Duong Xuan Nui, Dao Xuan Viet, Nguyen Ba Duc, Nguyen Duc Trung Kien

\section{Article info}

Recieved:

18/02/2020

Accepted:

$10 / 3 / 2020$

Keywords:

Materials science;

Monte Carlo simulation;

phase transition;

magnetic materials;

$q$-state clock model.

\begin{abstract}
We study the phase transition properties of the two-dimentional $q$-state clock model by an extensive Monte Carlo simulation. Several independent physical quantities, including the specific heat, the magnetization and the helicity modulus had been calculated for $q>5$. The specific heat and the magnetization shows the behavior of two distinct phase transitions, including the higher phase transition, $T_{2}$, between the disordered phase and the quasi-long-range ordered phase and the lower phase transition, $T_{1}$, between the quasi-long-range ordered phase and the quasi-long-range ordered phase. The helicity modulus not only show that $T_{2}$ is the Kosterlitz-Thouless phase transition but also has signature of phase transition around $T_{1}$.
\end{abstract}

